\title{
Elastic instabilities at a sliding interface
}

\author{
B. N. J. Persson \\ IFF, FZ Jülich, D-52425 Jülich, Germany \\ (Received 25 August 2000; revised manuscript received 20 November 2000; published 6 February 2001)
}

\begin{abstract}
I consider a semi-infinite elastic solid sliding on a flat hard substrate. I present a linear instability analysis to determine when the steady sliding motion becomes unstable with respect to infinitesimal perturbations. I consider a general case where the interfacial frictional shear stress depends not only on the sliding velocity but also on a state variable. I show that when the pressure in the contact area between the solids is constant, no linear instability occurs if the kinetic friction coefficient increases monotonically with the sliding velocity, $d \mu_{\mathrm{k}} / d v_{0}>0$. However, when the pressure at the interface varies spatially, elastic instabilities may also occur when $d \mu_{\mathrm{k}} / d v_{0}>0$. I discuss the physical origin of this effect, and suggest that these instabilities may be precursors of the Schallamach waves.
\end{abstract}

DOI: 10.1103/PhysRevB.63.104101

PACS number(s): $62.20 .-\mathrm{x}, 46.55 .+\mathrm{d}$

\section{INTRODUCTION}

During the sliding of a block on a substrate, rapid processes usually occur at the sliding interface independent (or weakly dependent) of the velocity $v_{0}$ of the center mass velocity of the block. These rapid processes can occur on many different length scales, starting at molecular distances (nanometers). ${ }^{1}$ For elastically soft materials such as rubber or gelatine, elastic instabilities have been observed on a macroscopic length scale. ${ }^{2-7}$ The most well-known instability for rubberlike materials is the so-called Schallamach waves. These wavelike instabilities have been observed for smooth rubber surfaces sliding on hard smooth substrates. The instabilities occur mainly for elastically soft rubber (not reinforced with carbon black), at high a enough sliding speed, where the rubber surface in front of the asperity undergoes a buckling that produces detachment waves. These are small regular folds filled with air, which cross the area of contact at velocities significantly greater than the imposed velocity $v_{0}$, from the compressive front zone to the tensile back zone. They move like wrinkles in a carper. In these circumstances, true sliding does not occur; folds are formed in the rubber and these provide relative motion between the two surfaces in adhesive contact. Based on experimental observation, in a rough picture we may consider every (Schallamach) instability wave as two crack tips that propagate in the same direction and with the same velocity: an opening crack (peeling) in the front region, and a closing mode (readhering) at the rear.

Elastic instabilities usually occur when the kinetic friction coefficient $\mu_{\mathrm{k}}$ decreases with increasing sliding velocity $v_{0}{ }^{1,8-11}$ However, the Schallamach waves are observed when $d \mu_{\mathrm{k}} / d v_{0}>0 .^{2-5}$ This is illustrated in Fig. 1, which shows experimental results for rubber sliding on (a) a glass substrate, and (b) a Teflon substrate. In (a), no Schallamach waves were detected, but stick-slip was observed when $d \mu_{\mathrm{k}} / d v_{0}<0$ (dashed line). On the other hand, in case (b), steady sliding is replaced with Schallamach wave propagation when the kinetic friction coefficient has reached $\mu_{\mathrm{c}}$ $\approx 2.2$. The Schallamach waves can be considered as a stress relieving mechanism that limit the buildup of friction with speed.
I note that the bell-shaped form of $\mu_{\mathrm{k}}$ in Fig. 1(a) is due to the internal friction of the rubber, which is particularly large when the perturbing frequencies are located in the transition zone between the rubbery region and the glassy region. ${ }^{1,12}$ By changing the rubber glass transition temperature (which depends on the molecular composition of the rubber) it is possible to shift the bell-shaped curve along the velocity axis by many orders of magnitude. ${ }^{1,12,13}$ This important fact can be used when choosing a rubber (for a particular application), in order to avoid interfacial stick-slip motion at the operating sliding velocities (see below).

Interfacial stick-slip motion may occur on many different length and time scales. If (for a finite-sized system) the frequency of a stick-slip instability happens to be close to a mechanical resonance (eigenmode) of the system, one may expect a strong coupling between the stick-slip motion and the mechanical resonance, which may result in a highly excited resonance mode. This may have severe mechanical consequences (devise failure), and is usually accompanied by a loud noise. ${ }^{14}$ The effect of stick-slip instabilities on the performance of rubber devices has been studied in great detail, e.g., in the context of water pump seals or ship propeller staves. Here, "squealing" (harmonic oscillations) is often observed, as a result of one of the natural frequencies of the system being preferentially excited. ${ }^{14}$ The resulting vibrations may be so large that detachment occurs in some interfacial areas. In these cases, the selection of a new elastomer with a different glass transition temperature may remove the stick-slip instabilities (by shifting them to a velocity region outside the one at which the devise normally operate), see above.

It is often believed that stick-slip can be removed by lubricating the sliding interface. However, in practice, the opposite is often observed. Thus, for example, shoes will often make noise when wet or slightly damp. The reason for this is that stick-slip usually has nothing directly to do with the magnitude of the kinetic friction coefficient (which may be strongly reduced upon lubrication), but rather, occurs when $d \mu_{\mathrm{k}} / d v_{0}<0$. Now, it is easy to understand why $d \mu_{\mathrm{k}} / d v_{0}$ may be negative when rubber is slid on a lubricated surface: At a low sliding speed, the lubrication fluid may be nearly 

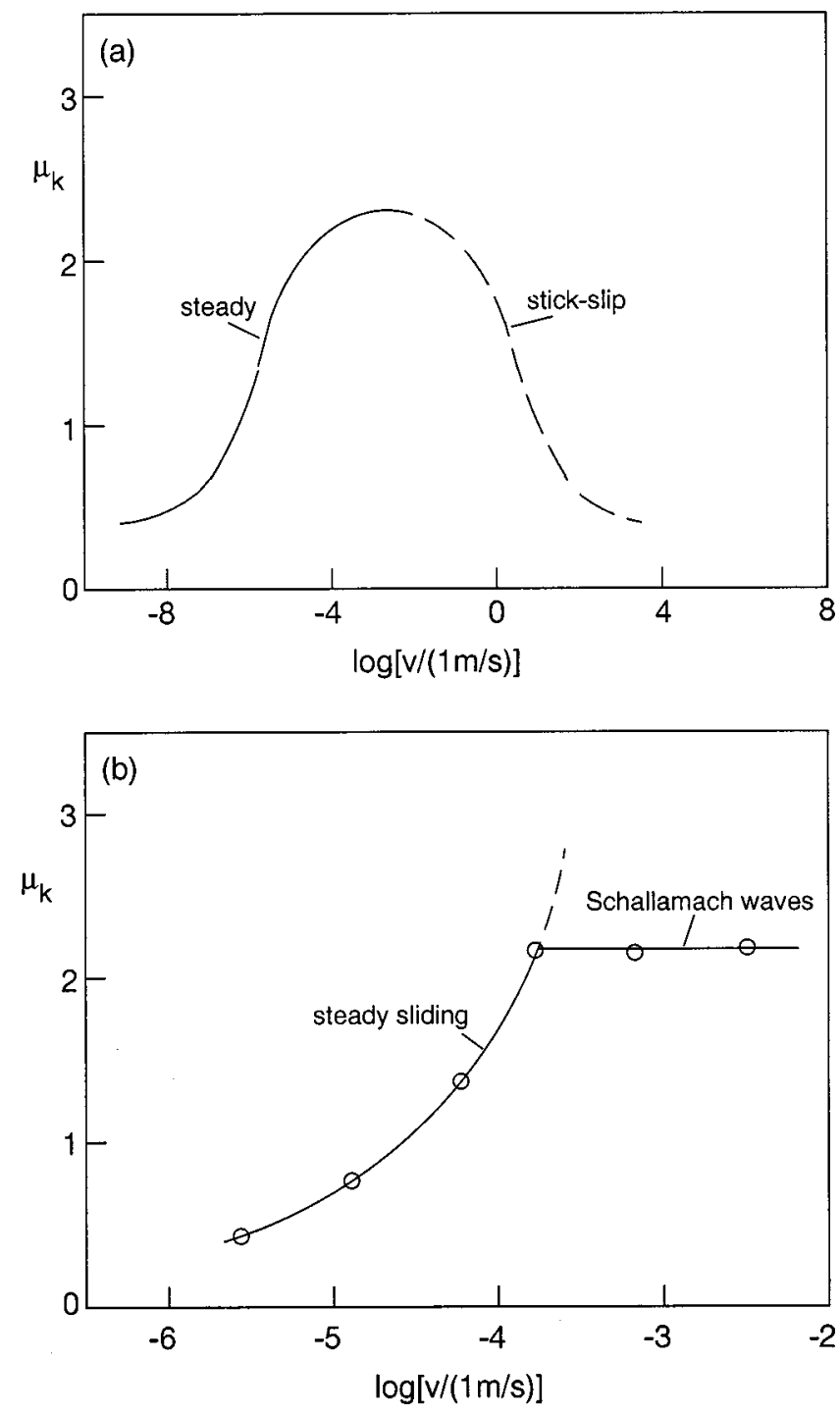

FIG. 1. (a) The kinetic friction coefficient for a rubber block sliding on a glass surface. In the dashed part of the friction curve, stick-slip motion is observed. (b) The kinetic friction coefficient for a rubber block sliding on a Teflon surface. Steady sliding is observed as long as $\mu_{\mathrm{k}}$ is below some critical value $\mu_{\mathrm{c}}$, while sliding occurs via the propagation of Schallamach waves when $\mu_{\mathrm{k}}$ reach $\mu_{\mathrm{c}}$. The figure is based on experimental data presented in Refs. 20 and 21 .

completely squeezed out from the rubber-substrate contact area, resulting in a large friction, while at a high-sliding velocity, a relative thick fluid layer remains trapped between the surfaces. Thus, the kinetic friction coefficient is likely to decrease with increasing sliding velocity $v_{0}$.

In this paper I consider a semi-infinite elastic solid sliding on a flat hard substrate. I present a linear instability analysis to determine when the steady sliding motion becomes unstable with respect to infinitesimal perturbations. I consider a general case where the interfacial frictional shear stress depends not only on the sliding velocity (in the $x$ direction) $v_{x}(x, t)$ but also on a state variable $\phi(x, t)$. I show that when the pressure $p_{0}(x)$ in the contact area between the solids is

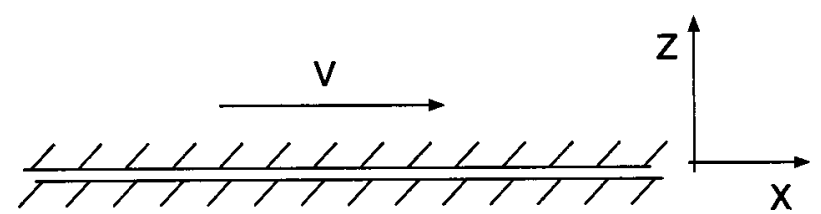

FIG. 2. Semi-infinite elastic solid $(z>0)$ sliding on a hard substrate $(z<0)$.

constant, no linear instability occurs if $d \mu_{\mathrm{k}} / d v_{0}>0$, but only when $d \mu_{\mathrm{k}} / d v_{0}<0$. However, when the pressure $p_{0}(x)$ varies with $x$ (which can be realized by squeezing an elastic body with a slightly curved surface towards the flat substrate surface), elastic instabilities may also occur for $d \mu_{\mathrm{k}} / d v_{0}$ $>0$. We discuss the physical origin of this effect, and suggest that these instabilities may be precursors of the Schallamach waves. I emphasize that although the theory presented in this paper was illustrated above with rubber friction, the theory is of general applicability, valid for any elastic solid. However, for elastically stiff materials, interfacial stick-slip instabilities may in practice only occur on such a large length scale that it cannot be observed in normalsized samples. But sometimes the physical systems of interest are very large, e.g., earthquake faults, and in these cases the theory presented below should be applicable. Finally, let me note that Adams has studied self-excited oscillations of two elastic half spaces sliding with a constant coefficient of friction. The present paper is a generalization of Ref. 15 in that we allow for a general velocity and state-dependent friction law, as well as for a spatially varying contact pressure $p_{0}(x)$.

\section{CONSTANT CONTACT PRESSURE $p_{0}=$ const}

Consider a semi-infinite elastic solid sliding on a flat rigid substrate. (In principle, we could allow the substrate and the elastic solid to have small wavelength surface roughness, but in this case, we can only consider the system on length scales much larger than the longest wavelength component of the surface roughness.) Assume that the elastic solid occupies the half space $z>0$, and let $(x, y, z)$ be a coordinate system with the $x y$ plane on the surface $z=0$ of the solid, see Fig. 2. We denote the two-dimensional position vector in the $z=0$ plane with $\mathbf{x}=(x, y)$. Assume that the stress $\sigma_{i}(\mathbf{x}, t)$ $=-\sigma_{i 3}(\mathbf{x}, 0, t)$ acts on the bottom surface of the elastic solid. We assume that the elastic block slides along the $x$ axis, and that $\sigma_{y}=0$. The frictional stress is assumed to depend on $x$ and on time $t$ and satisfies

$$
\sigma_{x}(x, t)=-\mu\left(v_{x}, \phi\right) \sigma_{z}(x, t)
$$

where the friction coefficient $\mu$ depends on the local sliding velocity $v_{x}(x, t)$ and on the state variable $\phi(x, t)$. Let us use a linear stability analysis to determine when the steady sliding motion becomes unstable. We write 


$$
\begin{aligned}
& v_{x}=v_{0}+\delta v_{x} \\
& \phi=\phi_{0}+\delta \phi,
\end{aligned}
$$

where $v_{0}$ and $\phi_{0}$ are independent of $x$ and $t$. We write the perturbations

$$
\delta v_{x}=\xi e^{i(\mathbf{q} \cdot \mathbf{x}-\omega t)}, \quad \delta \phi=\eta e^{i(\mathbf{q} \cdot \mathbf{x}-\omega t)},
$$

where $\xi$ and $\eta$ are small numbers. Similarly, we expand

$$
\begin{gathered}
\sigma_{z}=\sigma_{z}^{0}+\delta \sigma_{z} \equiv p_{0}+\delta \sigma_{z}, \\
\sigma_{x}=\sigma_{x}^{0}+\delta \sigma_{x} .
\end{gathered}
$$

Substituting Eqs. (2)-(6) in Eq. (1) and expanding $\mu$ to linear order in $\delta v_{x}$ and $\delta \phi$ gives:

$$
\sigma_{x}^{0}+\delta \sigma_{x}=-\left(\mu_{\mathrm{k}}\left(v_{0}\right)+\frac{\partial \mu}{\partial v_{x}} \delta v_{x}+\frac{\partial \mu}{\partial \phi} \delta \phi\right)\left(p_{0}+\delta \sigma_{z}\right)
$$

where $\mu_{\mathrm{k}}\left(v_{0}\right)=\mu\left[v_{0}, \phi_{0}\left(v_{0}\right)\right]$. Thus,

$$
\begin{gathered}
\sigma_{x}^{0}=-\mu_{\mathrm{k}}\left(v_{0}\right) p_{0} \\
\delta \sigma_{x}=-\mu_{\mathrm{k}}\left(v_{0}\right) \delta \sigma_{z}-\left(\frac{\partial \mu}{\partial v_{x}} \delta v_{x}+\frac{\partial \mu}{\partial \phi} \delta \phi\right) p_{0} .
\end{gathered}
$$

Using the theory of elasticity (assuming an isotropic elastic media for simplicity), one can calculate the displacement field $\mathbf{u}$ on the surface $z=0$ in response to the stress distributions $\delta \sigma_{x}$ and $\delta \sigma_{z}$. We have

$$
\delta u_{i}(\mathbf{q}, \omega)=M_{i j}(\mathbf{q}, \omega) \delta \sigma_{j}(\mathbf{q}, \omega)
$$

or, in matrix form,

$$
\delta \mathbf{u}(\mathbf{q}, \omega)=M(\mathbf{q}, \omega) \delta \sigma(\mathbf{q}, \omega)
$$

where the matrix ${ }^{16}$

$$
\begin{aligned}
M= & -\frac{i}{\rho c_{T}^{2}}\left(\frac{1}{P(q, \omega)}[Q(k, \omega)(\hat{z} \mathbf{q}-\mathbf{q} \hat{z})\right. \\
& \left.\left.+\left(\frac{\omega}{c_{T}}\right)^{2}\left(p_{L} \hat{z} \hat{z}+p_{T} \hat{q} \hat{q}\right)\right]+\frac{1}{p_{T}} \mathbf{e e}\right),
\end{aligned}
$$

where $\hat{q}=\mathbf{q} / q, \mathbf{e}=\hat{z} \times \hat{q}$ and where

$$
\begin{gathered}
P=\left(\frac{\omega^{2}}{c_{T}^{2}}-2 q^{2}\right)^{2}+4 q^{2} p_{T} p_{L}, \\
Q=2 q^{2}-\omega^{2} / c_{T}^{2}+2 p_{T} p_{L}, \\
p_{T}= \pm\left(\frac{\omega^{2}}{c_{T}^{2}} \pm i \epsilon-q^{2}\right)^{1 / 2}, \quad p_{L}= \pm\left(\frac{\omega^{2}}{c_{L}^{2}} \pm i \epsilon-q^{2}\right)^{1 / 2},
\end{gathered}
$$

where the + and - sign refers to $\omega>0$ and $\omega<0$, respectively, and where $\epsilon$ is an infinitesimal positive number. In the equations above, $\rho, c_{T}$, and $c_{L}$ are the mass density and the transverse and longitudinal sound velocities of the solid, respectively. In this paper, we will consider the sliding of an elastic solid (e.g., rubber) on a rigid flat surface. We assume that the two solids (for all times) are in contact over the whole $z=0$ plane so that $\delta u_{z}=0$ for $z=0$. We will also assume that $\delta \sigma_{y}=0$ so that Eq. (11) takes the form:

$$
\begin{gathered}
\delta u_{x}=M_{x x} \delta \sigma_{x}+M_{x z} \delta \sigma_{z}, \\
0=M_{z x} \delta \sigma_{x}+M_{z z} \delta \sigma_{z} .
\end{gathered}
$$

Equations (9) and (17) gives

$$
\delta \sigma_{x}=\frac{-p_{0}}{1-\mu_{\mathrm{k}}\left(v_{0}\right) M_{z x} / M_{z z}}\left(\frac{\partial \mu}{\partial v_{x}} \delta v_{x}+\frac{\partial \mu}{\partial \phi} \delta \phi\right) .
$$

Combining Eqs. (16) and (17) gives

$$
\delta \sigma_{x}=\frac{M_{z z} \delta u_{x}}{M_{x x} M_{z z}-M_{x z} M_{z x}} .
$$

Since $\delta v_{x}=-i \omega \delta u_{x}$, using Eqs. (18) and (19) gives

$$
\begin{aligned}
& \frac{M_{z z} \delta v_{x}}{M_{x x} M_{z z}-M_{x z} M_{z x}} \\
& =\frac{i \omega p_{0}}{1-\mu_{\mathrm{k}}\left(v_{0}\right) M_{z x} / M_{z z}}\left(\frac{\partial \mu}{\partial v_{x}} \delta v_{x}+\frac{\partial \mu}{\partial \phi} \delta \phi\right) .
\end{aligned}
$$

From Eq. (12) we get

$$
\begin{gathered}
M_{x x}=-\frac{i}{\rho c_{T}^{2}} \frac{p_{T}}{P}\left(\frac{\omega}{c_{T}}\right)^{2}, \\
M_{z z}=-\frac{i}{\rho c_{T}^{2}} \frac{p_{L}}{P}\left(\frac{\omega}{c_{T}}\right)^{2}, \\
M_{x z}=\frac{i}{\rho c_{T}^{2}} \frac{Q}{P} q_{x}, \\
M_{z x}=-\frac{i}{\rho c_{T}^{2}} \frac{Q}{P} q_{x} .
\end{gathered}
$$

Thus

$$
\begin{gathered}
\frac{M_{z z}}{M_{x x} M_{z z}-M_{x z} M_{z x}}=\frac{i \rho c_{T}^{2} P\left(\omega / c_{T}\right)^{2} p_{L}}{Q^{2} q^{2}+p_{L} p_{T}\left(\omega / c_{T}\right)^{4}}, \\
\frac{M_{z x}}{M_{z z}}=\frac{Q q_{x}}{p_{L}\left(\omega / c_{T}\right)^{2}} .
\end{gathered}
$$

If we write $\omega=i \alpha c_{T} q$ (where $q=\left|q_{x}\right|$ ) and $\kappa=c_{T} / c_{L}$ $=[(1-2 \nu) /(2-2 \nu)]^{1 / 2}($ where $\nu$ is the Poison ratio) we get

$$
\frac{M_{z z}}{M_{x x} M_{z z}-M_{x z} M_{z x}}=\rho c_{T}^{2} \alpha q G(\alpha)
$$




$$
\begin{gathered}
G(\alpha)=\frac{\alpha\left(\kappa^{2} \alpha^{2}+1\right)}{\alpha^{4}\left(\alpha^{2}+2\right)^{2}\left(\kappa^{2} \alpha^{2}\right.} \\
\frac{M_{z x}}{M_{z z}}=i H(\alpha) \operatorname{sgn}\left(q_{x}\right), \\
H(\alpha)=\frac{2+\alpha^{2}-2\left(\alpha^{2}+1\right)^{1 / 2}\left(\kappa^{2} \alpha^{2}+1\right)^{1 / 2}}{\alpha^{2}\left(\kappa^{2} \alpha^{2}+1\right)^{1 / 2}},
\end{gathered}
$$

where $\operatorname{sgn}\left(q_{x}\right)=q_{x} / q=1$ if $q_{x}>0$ and -1 if $q_{x}<0$. Note that

$$
G(\alpha) \rightarrow \frac{2}{\alpha\left(1+\kappa^{2}\right)} \quad \text { and } H(\alpha) \rightarrow-\kappa^{2}
$$

as $\alpha \rightarrow 0$. Using Eqs. (20), (27), and (29), we can write

$$
G(\alpha) \delta v_{x}=\frac{-p_{0} /\left(\rho c_{T}\right)}{1-i H(\alpha) \mu_{\mathrm{k}}\left(v_{0}\right) \operatorname{sgn}\left(q_{x}\right)}\left(\frac{\partial \mu}{\partial v_{x}} \delta v_{x}+\frac{\partial \mu}{\partial \phi} \delta \phi\right)
$$

The state variable $\phi$ is assumed to satisfy $y^{1,8,17}$

$$
\frac{\partial \phi}{\partial t}=1-v_{x} \phi / D
$$

Here, $D$ is a microscopic distance with which the two surfaces must move relative to each other in order to break the (local) "bond" between the surfaces. For example, if chain interdiffusion occurs at the interface, then $D$ will be some fraction of the chain length. Note that when $v_{x}=0$ (stationary contact) this equation gives $\phi=t$, i.e., $\phi$ equals the time of stationary contact. On the other hand, for uniform sliding, $v_{x}=$ const, Eq. (33) gives $\phi=D / v_{x}$, which is the average time a junction survives before being broken by the sliding motion. Using Eq. (33) gives

$$
-i \omega \eta=1-\left(v_{0}+\xi\right)\left(\phi_{0}+\eta\right) / D
$$

or

$$
\begin{gathered}
v_{0} \phi_{0}=D \\
-i \omega \eta=-\xi \phi_{0} / D-v_{0} \eta / D .
\end{gathered}
$$

Thus

$$
\eta=\frac{-\xi D / v_{0}^{2}}{1+\alpha q D c_{T} / v_{0}}
$$

or

$$
\delta \phi=\frac{-\delta v_{x} D / v_{0}^{2}}{1+\alpha q D c_{T} / v_{0}} .
$$

Combining Eqs. (32) and (38) gives

$$
G(\alpha)=\frac{-p_{0} /\left(\rho c_{T} v_{0}\right)}{1-i H(\alpha) \mu_{\mathrm{k}}\left(v_{0}\right) \operatorname{sgn}\left(q_{x}\right)}\left(a+\frac{b}{1+\alpha q D c_{T} / v_{0}}\right)
$$

where

$$
a=v_{0} \frac{\partial \mu}{\partial v_{x}}, \quad b=-\frac{D}{v_{0}} \frac{\partial \mu}{\partial \phi},
$$

where the partial derivatives are evaluated for $v_{x}=v_{0}$ and $\phi=\phi_{0}=D / v_{0}$. Now, let us first consider very small sliding velocities $v_{0}$. This case is equivalent to the small $\alpha$ limit where $G(\alpha) \approx 2 /\left[\alpha\left(1+\kappa^{2}\right)\right]$ and $H(\alpha) \approx-\kappa^{2}$. Substituting these results in Eq. (39) gives

$$
\frac{2}{\alpha\left(1+\kappa^{2}\right)}=\frac{-p_{0} /\left(\rho c_{T} v_{0}\right)}{1+i \kappa^{2} \mu_{\mathrm{k}}\left(v_{0}\right) \operatorname{sgn}\left(q_{x}\right)}\left(a+\frac{b}{1+\alpha q D c_{T} / v_{0}}\right) .
$$

Replacing $\alpha=-\mathrm{i} \omega / c_{T} q$ we get

$$
A(\omega) p_{0}=q,
$$

where

$$
A(\omega)=\frac{\mathrm{i} \omega\left(1+\kappa^{2}\right) /\left(2 \rho c_{T}^{2} v_{0}\right)}{1+i \kappa^{2} \mu_{k}\left(v_{0}\right) \operatorname{sgn}\left(q_{x}\right)}\left(a+\frac{b}{1-i \omega D / v_{0}}\right) .
$$

Equation (42) is a second-order equation in $\alpha$ that is easy to solve:

$$
\alpha=-\frac{1}{2}\left(\frac{a+b}{a \bar{q}}-\frac{1}{\lambda a}\right) \pm\left[\frac{1}{4}\left(\frac{a+b}{a \bar{q}}-\frac{1}{\lambda a}\right)^{2}-\frac{1}{\lambda a \bar{q}}\right]^{1 / 2}
$$

where $\bar{q}=q D c_{T} / v_{0}$ and

$$
\lambda=\frac{\left(1+\kappa^{2}\right) p_{0} /\left(\rho c_{T} v_{0}\right)}{2\left[1+i \kappa^{2} \mu_{k} \operatorname{sgn}\left(q_{x}\right)\right]} .
$$

The steady sliding state is unstable when $\operatorname{Im} \omega>0$ or $\operatorname{Re} \alpha$ $>0$. Thus, the boundary line in the $\left(q, v_{0}\right)$ plane separating the uniform sliding state from the stick-slip region is determined by the condition $\operatorname{Re} \alpha=0$, which gives

$$
\operatorname{Re}\left(\frac{a+b}{a \bar{q}}-\frac{1}{\lambda a}\right)=0
$$

or

$$
a+b=-\frac{q D E / p_{0}}{\left(1+\kappa^{2}\right)(1+\nu)},
$$

where $E=2 \rho c_{T}^{2}(1+\nu)$ is the elastic modulus. But since 


$$
\frac{d \mu_{\mathrm{k}}\left(v_{0}\right)}{d \ln v_{0}}=v_{0} \frac{d \mu\left(v_{0}, D / v_{0}\right)}{d v_{0}}=v_{0} \frac{\partial \mu}{\partial v_{x}}+v_{0} \frac{\partial \mu}{\partial \phi} \frac{d \phi_{0}}{d v_{0}}=a+b
$$

we get

$$
\frac{d \mu_{k}}{d \ln v_{0}}=-\frac{q D E / p_{0}}{\left(1+\kappa^{2}\right)(1+\nu)} .
$$

Let us now consider three different cases.

A. State-independent friction. Let us first assume that the friction coefficient only depends on the instantaneous sliding velocity $v_{x}$ :

$$
\mu\left(v_{x}, \phi\right)=\mu\left(v_{x}\right) .
$$

In this case, Eq. (46) reduces to $\mu_{\mathrm{k}}^{\prime}\left(v_{0}\right)=0$. Note that this equation is independent of $q$. Thus, stick-slip instabilities will occur at the sliding interface in those vertical strips in the $\left(v_{0}, q\right)$ plane where $\mu_{\mathrm{k}}^{\prime}\left(v_{0}\right)<0$, while steady motion occurs when $\mu_{\mathrm{k}}^{\prime}\left(v_{0}\right)>0$. This is illustrated in Fig. 3(a) for a typical case where there is only one stick-slip region at "low" sliding velocities.

B. State- and rate-dependent friction I. We assume, ${ }^{1,8,17}$

$$
\mu\left(v_{x}, \phi\right)=\mu_{k}^{0}\left(B_{\|} \ln v_{x}+B_{\perp} \ln \phi\right) .
$$

This friction law has been found to describe the low-velocity behavior of many systems of practical importance, e.g., stone sliding on stone, or paper sliding on paper. The first term on the right-hand side (RHS) of Eq. (51) describes the velocity dependence of the kinetic shear stress at low-sliding velocities (creep), while the second state-dependent term can have different origins, e.g., resulting from an increase in the contact area (due to perpendicular creep) with increasing time of stationary contact, or it may result from the (thermally activated) formation of capillary bridges between microscopically close but noncontacting surface asperities. Using Eq. (51) gives

$$
\frac{d \mu_{\mathrm{k}}}{d \ln v_{0}}=\mu_{\mathrm{k}}^{0}\left(B_{\|}-B_{\perp}\right)
$$

and Eq. (49) takes the form

$$
B_{\perp}-B_{\|}=\frac{q D E / p_{0}}{\mu_{\mathrm{k}}^{0}\left(1+\kappa^{2}\right)(1+\nu)} .
$$

Since $B_{\perp}$ and $B_{\|}$are assumed to be velocity independent, stick-slip instabilities will occur for $q<q_{c}$ [where $q_{c}$ is determined by Eq. (53)], while steady sliding occurs for $q$ $>q_{c}$. The dynamical phase diagram is shown in Fig. 3(b), where we have taken into account that the kinetic friction coefficient always increases at high enough velocity $v_{0}$, which gives rise to a high $v_{0}$ cutoff in the stick-slip region [this effect is not included in the friction law (51) that only can describe the low velocity creep behavior]. Thus, if the sliding body has a linear size $\sim L<1 / q_{c}$, no interfacial stickslip motion will occur. As an application, let us consider the recent experimental study of sliding friction of elastomer on a rough hard glass surface. ${ }^{7}$ In this case, it was observed that
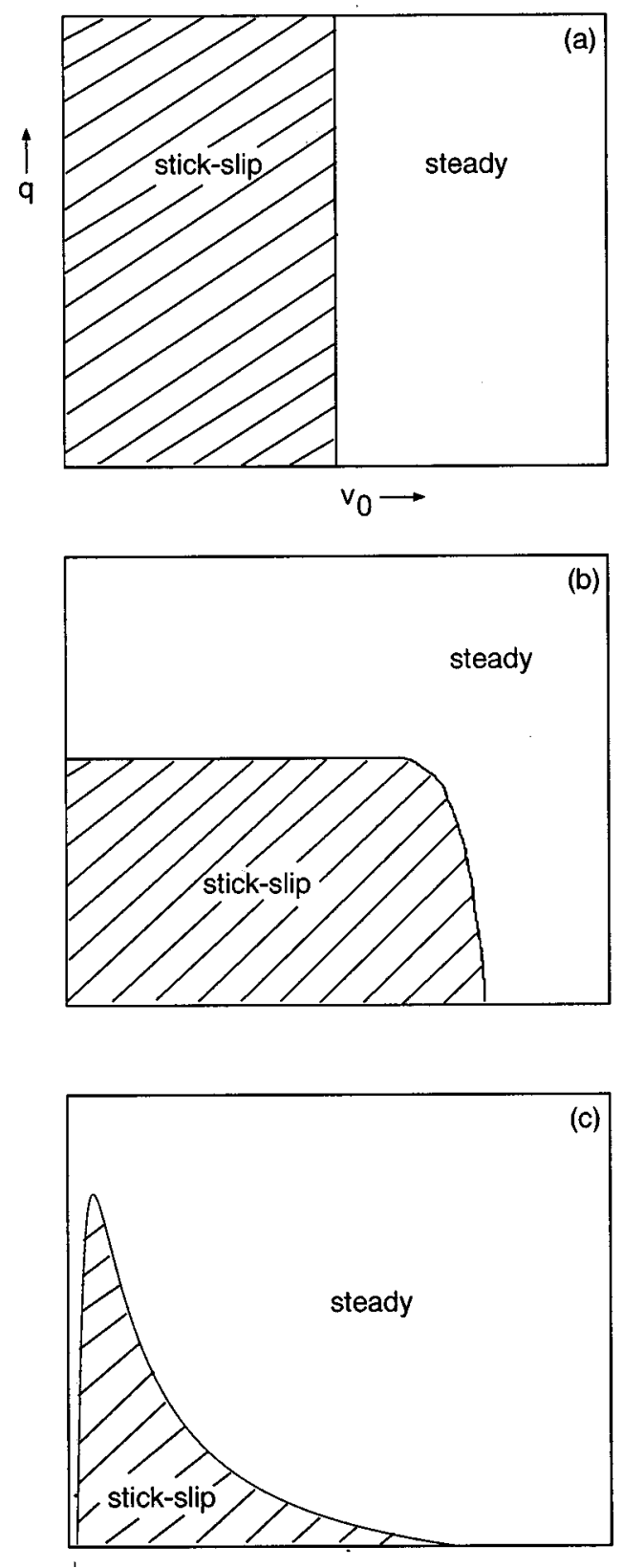

FIG. 3. Schematic dynamical phase diagrams (a)-(c) for three different friction laws. The dahed areas denote the region in the $\left(v_{0}, q\right)$ plane where nonsteady (stick-slip) motion occurs at the interface. $v_{0}$ is the sliding velocity far away from the interface, and $q$ the wave vector associated with the perturbation of the steady sliding motion. (a) is for the state-independent friction law [see Eq. (50)], while (b) and (c) are for the state-dependent friction laws (51) and (54). In (b) we have taken into account that the kinetic friction coefficient always increases at high enough velocity $v_{0}$, which gives rise to a high $v_{0}$-cutoff in the stick-slip region [this is not included in the friction law (51) that can only describe the low velocity (creep) behavior].

the sliding dynamics of the rubber block could be relatively well-described by the friction law (51) with $D \approx 1 \mu \mathrm{m}$ and $B_{\perp}-B_{\|} \sim 0.006-0.044$ depending on the temperature $T$. The (average) pressure $p_{0} \approx 0.1 \mathrm{MPa}$ and if we use the 
low-frequency elastic modulus $E \approx 3 \mathrm{MPa}$ in Eq. (53) we conclude that interfacial stick-slip instabilities, with the wavelength $\lambda$ larger than $\lambda_{\mathrm{c}}=2 \pi / q_{\mathrm{c}}=0.1-1 \mathrm{~cm}$, should occur at the sliding interface. However, the rubber block in the experiment was only $0.28 \mathrm{~cm}$ thick, which is comparable to $\lambda_{\mathrm{c}}$, while the calculation is for a semi-infinite rubber block. No information related to interfacial instabilities was presented in Ref. 7.

C. State- and rate-dependent friction II. We now consider a model where the static friction force increases with the time of stationary contact because of interdiffusion. In the simplest possible case one assumes that the increase in the static friction force is characterized by a single relaxation time $\tau$ and write, ${ }^{1,11}$

$$
\mu\left(v_{x}, \phi\right)=\mu_{k}^{0}\left[B_{\|} \ln v_{x}+C\left(1-e^{-\phi / \tau}\right)\right] .
$$

The friction dynamics that result from this friction law has been found to be in good agreement with the experiment for mica surfaces covered with chain molecules, (see Ref. 1 and Refs. 18 and 19). From Eq. (54)

$$
\frac{d \mu_{\mathrm{k}}}{d \ln v_{0}}=\mu_{\mathrm{k}}^{0}\left(B_{\|}-\frac{C D}{v_{0} \tau} e^{-D / v_{0} \tau}\right),
$$

and Eq. (49) takes the form

$$
C e^{-D / v_{0} \tau}\left(D / v_{0} \tau\right)-B_{\|}=\frac{q D E / p_{0}}{\mu_{\mathrm{k}}^{0}\left(1+\kappa^{2}\right)(1+\nu)} .
$$

This boundary line separating stick-slip from steady sliding is shown in Fig. 3(c). If $q=q\left(v_{0}\right)$ denotes the equation for the boundary line, the maximum $q$ for which stick-slip instabilities are possible is determined by $q^{\prime}\left(v_{0}\right)=0$ giving $v_{0}$ $=D / \tau$ and $q=p_{0}\left(1+\kappa^{2}\right)(1+\nu) / D E e$, where $e=2.718$.

\section{SPATIALLY VARYING CONTACT PRESSURE

$$
p_{0}=p_{0}(x)
$$

Let us now assume that the pressure distribution $p$ $=p(x)$ varies with $x$. It is easy to generalize the study presented above to get the following integral equation for $\delta u_{x}$ $=f(x)$ :

$$
\begin{aligned}
& \frac{1}{2 \pi} \int d x^{\prime}\left(\frac{1}{x-x^{\prime}+i \epsilon}+\frac{1}{x-x^{\prime}-i \epsilon}\right) f^{\prime}\left(x^{\prime}\right) \\
& -A(\omega) p_{0}(x) f(x)=0
\end{aligned}
$$

where, using that for rubberlike materials $\nu \approx 0.5$ and hence $\kappa \approx 0$ :

$$
A(\omega)=\frac{i \omega}{2 \rho c_{T}^{2}}\left(\frac{\partial \mu}{\partial v_{x}}-\frac{\partial \mu}{\partial \phi} \frac{D / v_{0}^{2}}{1-i \omega D / v_{0}}\right)
$$

Let us first consider a constant pressure $p_{0}(x)=$ const. In this case, the solutions to Eq. (57) have the form

$$
f(x)=u_{1} e^{i q_{x} x}
$$

Substituting this in Eq. (57) gives

$$
A(\omega) p_{0}=\left|q_{x}\right|,
$$

which is identical to Eq. (42). Next, let us find a solution to Eq. (57) corresponding to a nonuniform pressure distribution. For example, if

$$
f(x)=u_{1} \ln \left(b^{2}+x^{2} / a^{2}\right),
$$

where $a$ and $b$ are positive numbers with $b>1$, then the strain

$$
f^{\prime}(x)=\frac{2 x u_{1} / a^{2}}{b^{2}+x^{2} / a^{2}}
$$

and since

$$
\begin{aligned}
& \frac{1}{2 \pi} \int d x^{\prime} \frac{2 x^{\prime} / a^{2}}{b^{2}+x^{\prime 2} / a^{2}}\left(\frac{1}{x-x^{\prime}+i \epsilon}+\frac{1}{x-x^{\prime}-i \epsilon}\right) \\
& \quad=-\frac{2 b / a}{b^{2}+x^{2} / a^{2}}
\end{aligned}
$$

Eq. (57) takes the form

$$
\frac{-2 b / a}{b^{2}+x^{2} / a^{2}}-A p_{0}(x) \ln \left(b^{2}+x^{2} / a^{2}\right)=0 .
$$

This equation is satisfied if we choose

$$
p_{0}(x)=\frac{2 P_{0} b^{2} \ln b}{\left(b^{2}+x^{2} / a^{2}\right) \ln \left(b^{2}+x^{2} / a^{2}\right)}
$$

with

$$
A P_{0}=-\frac{1}{a b \ln b} .
$$

Note that $p_{0}(0)=P_{0}$ and that $p_{0}(x)>0$ for all $x$, while $p_{0}(x) \rightarrow 0$ as $x \rightarrow \pm \infty$.

Since $a>0$ and $b>1$, Eqs. (58) and (66) predict that $\operatorname{Im} \omega>0$ if $d \mu_{\mathrm{k}} / d v_{0}>0$. Thus, the steady sliding state is unstable with respect to small perturbations when $d \mu_{\mathrm{k}} / d v_{0}$ $>0$, in sharp contrast to the case of a constant pressure $p(x)=p_{0}$ where steady sliding is unstable only when $d \mu_{\mathrm{k}} / d v_{0}<0$.

Let us calculate the pressure $p_{1}$ corresponding to the displacement field $\delta u_{x}=f(x)$. From Eqs. (16) and (17) we get

$$
\begin{aligned}
\delta \sigma_{z}\left(q_{x}\right) & =-\frac{M_{z x}}{M_{x x} M_{z z}-M_{x z} M_{z x}} \delta u_{x}\left(q_{x}\right) \\
& =\frac{2 \kappa^{2}}{1+\kappa^{2}} \rho c_{T}^{2}\left(i q_{x}\right) \delta u_{x}\left(q_{x}\right) .
\end{aligned}
$$

Thus, returning to real space,

$$
p_{1}(x)=\frac{2 \kappa^{2}}{1+\kappa^{2}} \rho c_{T}^{2} f^{\prime}(x)=\frac{2 \kappa^{2}}{1+\kappa^{2}} \rho c_{T}^{2} \frac{2 x u_{1} / a^{2}}{b^{2}+x^{2} / a^{2}} .
$$


Note that while $p_{0}(x)$ is symmetric around $x=0, p_{1}(x)$ changes sign as $x \rightarrow-x$. Since $p_{1}$ decays slower with increasing $|x|$ than $p_{0}(x)$, the total pressure $p(x)=p_{0}(x)$ $+p_{1}(x)$ will be negative for some $x$. Within the present model, this indicates that, for arbitrary low sliding velocity (assuming $d \mu_{\mathrm{k}} / d v_{0}>0$ ), detachment will occur in some interfacial region. However, for elastically soft materials such as rubber, adhesion is very important, and a large enough negative pressure is necessary in order to break the rubbersubstrate bond. Thus, for real systems, detachment will occur only for a high enough sliding velocity. A more complete analysis of the detachment process requires an extension of the model above to take into account the adhesion; in this case it would be very interesting to perform a nonlinear instability analysis to the third order, in order to gain a deeper insight into the detachment process.

We note that the integral Eq. (57), for a given pressure $p_{0}(x)$, will, in general, have an infinite set of solutions ("eigenvectors") $f_{\nu}(x)$. In the present paper we have only studied one of these solutions. The other solutions may, or may not, be associated with eigenvalues with $\operatorname{Im} \omega_{\nu}>0$. If this inequality is satisfied, the steady sliding motion is unstable with respect to the perturbation $f_{\nu}(x)$.

\section{SUMMARY}

I have studied the sliding of a semi-infinite elastic solid on a flat hard substrate and determined when the steady sliding motion becomes unstable with respect to infinitesimal perturbations. I assumed that the interfacial frictional shear stress depends not only on the sliding velocity but also on a state variable. When the pressure in the contact area between the solids is constant, no linear instability occurs if the kinetic friction coefficient increases monotonically with the sliding velocity, $d \mu_{\mathrm{k}} / d v_{0}>0$. However, when the pressure at the interface varies spatially, elastic instabilities may also occur when $d \mu_{\mathrm{k}} / d v_{0}>0$. These instabilities may be precursors of the Schallamach waves.

\section{ACKNOWLEDGMENTS}

I thank T. Baumberger, C. Caroli, G. Heinrich, and O. Rosin for useful discussions and preprints, and BMBF for a grant related to the German-Israeli Project Cooperation "Novel Tribological Strategies from the Nano-to-Meso Scales." I also thank Pirelli Pneumatici for a research grant related to "Physical principles of rubber friction and application to tires."
${ }^{1}$ B. N. J. Persson, Sliding Friction: Physical Principles and Applications, 2nd ed. (Springer, Heidelberg, 2000).

${ }^{2}$ A. Schallamach, Wear 17, 301 (1971).

${ }^{3}$ A. D. Roberts and S. A. Jackson, Nature (London) 257, 118 (1975).

${ }^{4}$ S. P. Arnold, A. D. Roberts, and A. D. Taylor, J. Nat. Rubber Res. 2, 1 (1987).

${ }^{5}$ M. Barquins, Mater. Sci. Eng. 73, 45 (1985).

${ }^{6}$ H. R. Berger and G. Heinrich, KGK Kautschuk Gummi Kunststoffe 53, 200 (2000).

${ }^{7}$ O. Rosin and K. L. Coeyrehourcq (unpublished).

${ }^{8}$ T. Baumberger, P. Berthoud, and C. Caroli, Phys. Rev. B 60, 3928 (1999).

${ }^{9}$ M. G. Rozman, M. Urbakh, J. Klafter, and F. J. Elmer, J. Phys. Chem. 102, 7924 (1998).

${ }^{10}$ M. Porto, M. Urbakh, and J. Klafter, Europhys. Lett. 50, 326
(2000).

${ }^{11}$ B. N. J. Persson, Phys. Rev. B 55, 8004 (1997).

${ }^{12}$ B. N. J. Persson and E. Tosatti, J. Chem. Phys. 112, 2021 (2000).

${ }^{13}$ K. A. Grosch, Proc. R. Soc. London, Ser. A 274, 21 (1963).

${ }^{14}$ R. A. L. Rorrer, Rubber Chem. Technol. 73, 488 (2000).

${ }^{15}$ G. G. Adams, J. Appl. Mech. 62, 867 (1995).

${ }^{16}$ B. N. J. Persson, Phys. Rev. B 51, 13568 (1995); Surf. Sci. 457, 345 (2000).

${ }^{17}$ J. R. Rice and A. L. Ruina, J. Appl. Mech. 105, 343 (1983).

${ }^{18}$ B. N. J. Persson, Surf. Sci. Rep. 33, 83 (1999).

${ }^{19}$ H. Yoshizawa, Y.-L. Chen, and J. Israelachvili, Wear 168, 161 (1993).

${ }^{20}$ S. P. Arnold, A. D. Roberts, and A. D. Taylor, J. Nat. Rubber Res. 2, 1 (1987).

${ }^{21}$ A. D. Roberts, Rubber Chem. Technol. 65, 673 (1992). 